\title{
NEAREST POINTS TO CLOSED SETS AND DIRECTIONAL DERIVATIVES OF DISTANCE FUNCTIONS
}

\section{Simon FITZPATRICK}

We investigate the circumstances under which the distance function to a closed set in a Banach space having a one-sided directional derivative equal to 1 or -1 implies the existence of nearest points. In reflexive spaces we show that at a dense set of points outside a closed set the distance function has a directional derivative equal to 1 .

\section{INTRODUCTION}

Let $K$ be a closed nonempty subset of a Banach space $X$. The distance function

$$
d(x)=\inf \{\|x-z\|: z \in K\}
$$

is Lipschitzian of rank 1 so that for $\|y\|=1$ we have

$$
-1 \leqslant \liminf _{t \rightarrow 0+} \frac{d(x+t y)-d(x)}{t} \leqslant \limsup _{t \rightarrow 0+} \frac{d(x+t y)-d(x)}{t} \leqslant 1 .
$$

If the one-sided directional derivative

$$
d_{+}^{\prime}(x)(y)=\lim _{t \rightarrow 0+} \frac{d(x+t y)-d(x)}{t}
$$

exists, then $\left|d_{+}^{\prime}(x)(y)\right| \leqslant 1$ if $\|y\|=1$. In this note we investigate the circumstances under which $d_{+}^{\prime}(x)(\vec{x})$ can equal 1 or -1 for some unit vector $\vec{x}$.

As shown by our previous work $[4,5]$ and by Zajicek $[10]$, differentiability properties of $d$ are related to nonemptiness and continuity of the metric projection

$$
P(x)=\{z \in K:\|z-x\|=d(x)\} .
$$

In Section 2 we give a geometric condition on the Banach space $X$ and a unit vector $\vec{x}$ which is necessary and sufficient for $\left|d_{+}^{\prime}(x)(\vec{x})\right|=1$ to imply that $P(x)$ is nonempty. It is not possible to deduce continuity of $P$ at $x$ from $d_{+}^{\prime}(x)(\vec{x})=-1$ but if the norm is locally uniformly convex at $\vec{x}$ and $d_{+}^{\prime}(x)(\vec{x})=1$ then $P$ is continuous at $\boldsymbol{x}$.

In Section 3 we show that if $X$ is reflexive then there is a dense subset $D$ of $X \backslash K$ such that if $v \in D$ there is $\vec{v} \in X$ with $\|\vec{v}\|=1$ and $d_{+}^{\prime}(v)(\vec{v})=1$ and $d_{+}^{\prime}(v)(-\vec{v})=-1$.

\section{Received 17 May 1988}

The author would like to thank John Giles for suggesting this problem.

Copyright Clearance Centre, Inc. Serial-fee code: 0004-9729/89 \$A2.00+0.00. 


\section{EXISTENCE OF NEAREST POINTS}

If $P(x)$ is nonempty and $x \in X \backslash K$ then there is $\vec{x} \in X$ with $\|\vec{x}\|=1$ and $d_{+}^{\prime}(x)(\vec{x})=-1$ : simply take any $\vec{x}$ with $x+d(x) \vec{x} \in P(x)$. The following calculation is useful for constructing examples.

LеммA 2.1. If $\left\|y_{n}\right\|=1$ and $\lim _{n \rightarrow \infty}\left\|\vec{x}+y_{n}\right\|=2$ and $\|\vec{x}\|=1$ let $K=$ $\left\{z_{n}: b \in N\right\}$ where $z_{n}=\left(1+\frac{1}{n}\right)\left\|\vec{x}+y_{n}\right\|^{-1}\left(\vec{x}+y_{n}\right)$. Then $d_{+}^{\prime}(0)(\vec{x})=-1=$ $-d_{+}^{\prime}(0)(-\vec{x})$.

ProOF: If $\alpha_{N}=\left(1+\frac{1}{n}\right)\left\|\vec{x}+y_{n}\right\|^{-1}$, we have by convexity of the norm $t^{-1}\left(\left\|z_{n}-t \vec{x}\right\|-\left\|z_{n}\right\|\right) \leqq \alpha_{n}^{-1}\left(\left\|z_{n}-\alpha_{n} \vec{x}\right\|-\left\|z_{n}\right\|\right)$ whenever $1 \leqq \alpha_{n}$. Thus

$$
\begin{aligned}
-1 & \leqq \liminf _{t \rightarrow 0} \frac{d(t \vec{x})-d(0)}{t} \leqq \limsup _{t \rightarrow 0} \frac{d(t \vec{x})-d(0)}{t} \\
& =\limsup _{t \rightarrow 0} t^{-1} \inf _{n}\left(\left\|t \vec{x}-z_{n}\right\|-1\right) \\
& =\limsup _{t \rightarrow 0} t^{-1} \inf _{n}\left(\left\|z_{n}-t \vec{x}\right\|-\left\|z_{n}\right\|+\frac{1}{n}\right) \\
& \leqq \limsup _{n \rightarrow \infty} \alpha_{n}^{-1}\left(\left\|z_{n}-\alpha_{n} \vec{x}\right\|-\left\|z_{n}\right\|\right) \\
& =\lim _{n \rightarrow \infty}\left(\left\|y_{n}\right\|-\left\|\vec{x}+y_{n}\right\|\right)=-1
\end{aligned}
$$

so that $d_{+}^{\prime}(0)(\vec{x})=-1=-d_{+}^{\prime}(0)(-\vec{x})$.

TheOREM 2.2. Let $X$ be a Banach space and $\vec{x} \in X$ with $\|\vec{x}\|=1$. The following statements are equivalent:

(a) if $K$ is nonempty closed subset of $X$ and $x \in X \backslash K$ with $d_{+}^{\prime}(x)(\vec{x})=$ -1 then $x$ has a nearest point in $K$;

(b) if $K$ is a nonempty closed subset of $X$ and $x \in X \backslash K$ with $\liminf _{t \rightarrow 0+}(d(x+t \vec{x})-d(x)) / t=-1$ then $x$ has a nearest point in $K$;

(c) if $\left\|y_{n}\right\|=1$ and $\lim _{n \rightarrow \infty}\left\|\vec{x}+y_{n}\right\|=2$ then $\left(y_{n}\right)$ has a convergent subsequence.

Proof: Clearly (b) implies (a). Suppose (c) holds and, to prove (b), let $t_{n} \rightarrow 0+$ with $\lim _{n \rightarrow \infty}\left(d\left(x+t_{n} \vec{x}\right)-d(x)\right) / t_{n}=-1$. Choose $z_{n} \in K$ with $\left\|x+t_{n} \vec{x}-z_{n}\right\|<$ 


$$
\begin{aligned}
& d\left(x+t_{n} \vec{x}\right)+t_{n}^{2} . \text { Then } \\
& \quad\left(d\left(x+t_{n} \vec{x}\right)-d(x)\right) / t_{n}>\left(\left\|x+t_{n} \cdot \vec{x}-z_{n}\right\|-\left\|x-z_{n}\right\|\right) / t_{n}-t_{n} \\
& \geqq-t_{n}+\left(\left\|x-z_{n}\right\|-\left\|x-z_{n}-\right\| x-z_{n}\|\vec{x}\|\right) /\left\|x-z_{n}\right\|=-t_{n}+1-\left\|\vec{x}+y_{n}\right\|
\end{aligned}
$$

where $y_{n}=-\left\|x-z_{n}\right\|^{-1}\left(x-z_{n}\right)$. Thus $\left\|y_{n}\right\|=1$ and $\left\|\vec{x}+y_{n}\right\| \rightarrow 2$ so $\left(y_{n}\right)$ has a convergent subsequence $\left(y_{n_{j}}\right),\left(z_{n_{j}}\right)$ converges to a point $z \in K$ (since $K$ is closed) and $\|x-z\|=d(x)$. Finally suppose there is a sequence $\left(y_{n}\right)$ with $\left\|y_{n}\right\|=1$ and $\left\|\vec{x}+y_{n}\right\| \rightarrow 2$ but $\left(y_{n}\right)$ has no convergent subsequence. Then $K=\left\{\left(1+\frac{1}{n}\right)\left\|\vec{x}+y_{n}\right\|^{-1}\left(\vec{x}+Y_{n}\right): n \in N\right\}$ is a closed set and 0 has no nearext point in $K$. However Lemma 2.1 shows that $d_{+}^{\prime}(0)(\vec{x})=-1$, contradicting (a).

If $d_{+}^{\prime}(x)(\vec{x})=1$ and $\|\vec{x}\|=1$ we can get a similar result or we can show continuity of $P$ at $x$ under a stronger hypothesis. We say that $\left(z_{n}\right)$ is a minimising sequence for $x$ if $z_{n} \in K$ and $\lim _{n \rightarrow \infty}\left\|x-z_{n}\right\|=d(x)$.

Proposition 2.3. Suppose $x \in X \backslash K$ and $\vec{x} \in X$ with $\|\vec{x}\|=1$ and $\limsup _{t \rightarrow 0+}(d(x+t \vec{x})-d(x)) / t=1$. If $\left(z_{n}\right)$ is a minimising sequence for $x$ and $y_{n}=\left\|x-z_{n}\right\|^{-1}\left(x-z_{n}\right)$ then $\left\|\vec{x}+y_{n}\right\| \rightarrow 2$.

PROOF: Let $r_{n} \rightarrow 0+$ so that $\lim _{n \rightarrow \infty}\left(d\left(x+t_{n} \vec{x}\right)-d(x)\right) / t_{n}=1$. We may assume that $t_{n}<d(x)$ and $t_{n}^{2}>\left\|x-z_{n}\right\|-d(x)$. Now

$$
\begin{aligned}
t_{n}^{-1}(d(x+t \vec{x})-d(x)) & \leqslant t_{n}^{-1}\left(\left\|z+t_{n} \vec{x}-z_{n}\right\|-\left\|x-z_{n}\right\|+t_{n}^{2}\right) \\
& \leqq\left\|x-z_{n}\right\|^{-1}\left(\left\|x-z_{n}+\right\| x-z_{n}\|\vec{x}\|-\left\|x-z_{n}\right\|\right)+t_{n} \\
& =\left\|\vec{x}+y_{n}\right\|-1+t_{n} .
\end{aligned}
$$

Thus $\liminf _{n \rightarrow \infty}\left\|\vec{x}+y_{n}\right\|=2$. Since $\left\|\vec{x}+y_{n}\right\| \leqq 2$ we have $\left\|\vec{x}+y_{n}\right\| \rightarrow 2$.

We use this to get the analogue of Theorem 2.2 .

Theorem 2.4. Let $X$ be a Banach space and $\vec{x} \in X$ with $\|\vec{x}\|=1$. The following statements are equivalent:

(a) for each closed nonempty subset $K$ of $X$ and $x \in X \backslash K$, if $d_{+}^{\prime}(x)(\vec{x})=$ 1 then $K$ has a nearest point to $x$; 
(b) for each closed nonempty subset $K$ of $X$ and $x \in X \backslash K$,if $\limsup _{t \rightarrow 0+}(d(x+t \vec{x})-d(x)) / t=1$ then every minimising sequence for $x$ has a convergent subsequence;

(c) if $\left\|y_{n}\right\|=1$ and $\lim _{n \rightarrow \infty}\left\|\vec{x}+y_{n}\right\|=2$ then $\left(y_{n}\right)$ has a convergent subsequence.

Proof: Clearly (b) implies (a). Assume (c) and suppose $x \in X \backslash K$ with $\limsup _{t \rightarrow 0+}(d(x+t \vec{x})-d(x)) / t=1$. Then any minimising sequence $\left(z_{n}\right)$ has $\left\|\vec{x}+y_{n}\right\|$ $\rightarrow 2$ where $y_{n}=\left\|x-z_{n}\right\|^{-1}\left(x-z_{n}\right)$, by Proposition 2.3. Therefore $\left(y_{n}\right)$ has a convergent subsequence $\left(y_{n_{j}}\right)$ and $\left(z_{n_{j}}\right)$ is convergent because $\left\|x-z_{n}\right\| \rightarrow d(x)>0$.

Finally, suppose (c) does not hold so there are $y_{n}$ with $\left\|y_{n}\right\|=1$ and $\lim _{n \rightarrow \infty}$ $\left\|\vec{x}+y_{n}\right\|=2$ but $\left(y_{n}\right)$ has no convergent subsequence. Let $K=$ $\left\{-\left(1+\frac{1}{n}\right)\left\|\vec{x}+y_{n}\right\|^{-1}\left(\vec{x}+y_{n}\right): n \in N\right\}$. Then $K$ is closed and 0 has no nearest point in $K$. But Lemma 2.1 shows that $d_{+}^{\prime}(0)(\vec{x})=1$.

Recall that $X$ is locally uniformly convex at $\vec{x}$ with $\|\vec{x}\|=1$ provided every sequence $\left(y_{n}\right)$ with $\left\|y_{n}\right\|=1$ and $\left\|\vec{x}+y_{n}\right\| \rightarrow 2$ has $\left\|\vec{x}-y_{n}\right\| \rightarrow 0$.

Theorem 2.5. Let $X$ be a Banach space and $\vec{x} \in X$ with $\|\vec{x}\|=1$. The following statements are equivalent:

(a) for each nonempty closed set $K$ and $x \in X \backslash K$, if $d_{+}^{\prime}(x)(\vec{x})=1$ then $P(x)$ has exactly one element;

(b) for each nonempty closed set $K$ and $x \in X \backslash K$, if limsup $(d(x+t \vec{x})-d(x)) / t=1$ then every minimising sequence for $x$ converges to $x-d(x) \vec{x}$ and $P$ is continuous at $x$;

(c) $X$ is locally uniformly convex at $\vec{x}$.

Proof: Clearly (b) implies (a). Assume (c) and let $\limsup _{t \rightarrow 0+}(d(x+t \vec{x})-d(x)) / t$ $=1$ and $x \in X \backslash K$. Suppose $\left(z_{n}\right)$ is a minimising sequence for $x$. By Proposition 2.3, $y_{n}=\left\|x-z_{n}\right\|^{-1}\left(x-z_{n}\right)$ has $\left\|\vec{x}+y_{n}\right\| \rightarrow 2$. Since $\left\|y_{n}\right\|=1$ we have $\left\|\vec{x}-y_{n}\right\| \rightarrow 0$ so that $z_{n} \rightarrow x-d(x) \vec{x}$. The continuity of $P$ at $x$ follows immediately.

Finally, suppose $X$ is not locally uniformly convex at $\vec{x}$. Then there are $y_{n} \in K$ with $\left\|y_{n}\right\|=1$ and $\left\|\vec{x}+y_{n}\right\| \rightarrow 2$, but $\left\|\vec{x}-y_{n}\right\| \geqslant \delta>0$ for all $n$. If $\left(y_{n}\right)$ has no 
convergent subsequence we can use Theorem 2.4 to get a closed set $K$, and $x \in X \backslash K$ so that $d_{+}^{\prime}(x)(\vec{x})=1$ and $P(x)=\emptyset$. Otherwise some subsequence $\left(y_{n}\right)$ converges to a point $y$ of $X$ with $\|\vec{x}-y\| \geqslant \delta>0$ and $\|\vec{x}+y\|=2$. Since $\|y\|=1$ for $K=\{-\vec{x},-y\}$ we have $d_{+}^{\prime}(0)(\vec{x})=1$, but $P(0)=\{-\vec{x}, y\}$ contradicting (a).

We note that no geometric condition on the norm combined with $d_{+}^{\prime}(x)(\vec{x})=-1$ can give single-valuedness of the metric projection at $x$ : let $x=0$ and $K$ be the unit sphere of $X$, for example.

\section{DENSE SETS OF POINTS WITH ONE-SIDED DERIVATIVE 1 OR -1}

We start with an example to show that we need to consider reflexive Banach spaces.

Example 3.1. If $X$ is a nonreflexive Banach space, let $x^{*}$ be any element of $X^{*}$ such that $\left\|x^{*}\right\| \cdot\|x\|>x^{*}(x)$ for all $x \neq 0$. These exist by James' Theorem [7]. Then $K=\operatorname{ker} x^{*}$ is closed subset of $X$ with $\left|d_{+}^{\prime}(x)(y)\right|<1$ for all $x \in X \backslash K$ and $\|y\|=1$.

Indeed, we have $d(x)=\left|x^{*}(x)\right|$ for every $x \in X$ so that $d_{+}^{\prime}(x)(y)=$ $\operatorname{sgn}\left(x^{*}(x)\right) x^{*}(y)$.

Preiss [0] (see [1], p.523) has shown that any Lipschitzian function on a Banach space $X$ which is an Asplund space is Fréchet differentiable at a dense set of points of $X$.

Theorem 3.2. If $X$ is an Asplund space and $K$ is a closed nonempty subset of $X$ then there is a dense set of points in $X \backslash K$ at which $d$ is Fréchet differentiable with derivative having norm 1 .

Proof: Using Preiss' result we only need to show that if $d$ is Fréchet differentiable at a point $x \in X \backslash K$ then $\left\|d^{\prime}(x)\right\|=1$. But we showed this in [4], Theorem 2.6.

Corollary 3.3. If $X$ is a reflexive Banach space and $K$ is a closed subset of $X$ then there is a dense subset $D$ of $X \backslash K$ such that for each $x \in D$ there is $\vec{x} \in X$ with $\|\vec{x}\|=1, d_{+}^{\prime}(x)(\vec{x})=1$ and $d_{+}^{\prime}(x)(-\vec{x})=-1$.

Proof: Let $D$ be the dense set given in Theorem 3.2. For each $x \in D$ let $\vec{x}$ be any element with $\|\vec{x}\|=1$ and $d^{\prime}(x)(\vec{x})=1$.

This corollary together with Theorem 2.4 (or Theorem 2.2) and Theorem 2.5 show that in a reflexive space with Kadec norm there is a dense set $D$ of points in $X \backslash K$ such that each $x \in D$ has a nearest point $K$, and if the norm is locally uniformly convex then $P$ is continuous at each point of $D$. However Lau [8] has shown that the sets of points with those properties in such reflexive spaces are residual in $X \backslash K$. Thus we ask the following question. 
Problem 3.4. If $X$ is a reflexive Banach space and $K$ a closed subset of $X$. Is the set

$$
\left\{x \in X \backslash K:(\exists \vec{x} \in X) \quad\left((\|\vec{x}\|=1) \&\left(d_{+}^{\prime}(x)(\vec{x})=1\right)\right)\right\}
$$

residual in $X \backslash K$ ?

\section{REFERENCES}

[1] J.M. Borwein and D. Preiss, 'A smooth variational principle with applications to subdifferentiability of convex functions', Trans. Amer. Math. Soc. 303 (1987), 517-527.

[2] J.M. Borwein and J.R. Giles, 'The proximal normal formula in Banach spaces', Trans. Amer. Math. Soc. 302 (1987), 371-381.

[3] J.M. Borwein, S.P. Fitzpatrick and J.R. Giles, 'The differentiability of real valued functions on normed linear spaces using generalised subgradients', J. Math. Anal. Appl. 128 (1987), 512-534.

[4] S. Fitzpatrick, 'Metric projections and the differentiability of distance functions', Bull. Austral. Math. Soc. 22 (1980), 291-312.

[5] S. Fitzpatrick, 'Differentiation of real valued functions and continuity of metric projections', Proc. Amer. Math. Soc. 81 (1984), 544-548.

[a] J.R. Giles, 'A distance function property implying differentiability', Bull. Austral. Math. Soc. (to appear).

[7] R.C. James, 'Weak compactness and reflexivity', Israel Math. J. 2 (1964), 101-119.

[8] Ka Sing Lau, 'Almost Chebyshev subsets in reflexive Banach spaces', Indiana Univ. Math. J. 2 (1978), 791-795.

[9] D. Preiss, 'Differentiability of Lipschitz functions on Banach spaces', (submitted).

[10] L. Zajicek, 'Differentiability of distance functions and points of multivaluedness of the metric projection in Banach spaces', Czech. Math. J. 33 (1983), 292-308.

Department of Mathematics and Statistics

University of Auckland

Auckland,

New Zealand 